Benchmarks

\title{
Comparative analysis of mitosis-specific antibodies for bullk purification of mitotic populations by fluorescence-activated cell sorting
}

\author{
Amy E. Campbell ${ }^{1,2, *}$, Chris C.-S. Hsiung ${ }^{1,2, *}$, and Gerd A. Blobel ${ }^{1,2}$ \\ ${ }^{1}$ Division of Hematology, The Children's Hospital of Philadelphia, \\ Philadelphia, PA and ${ }^{2}$ Perelman School of Medicine, University of \\ Pennsylvania, Philadelphia, PA
}

${ }^{*}$ A.E.C and C.C.-S. H. contributed equally to this work.

BioTechniques 56:90-94 (February 2014) doi 10.2144/000114137

Keywords: Mitosis; FACS; H3S10ph; MPM2

Supplementary material for this article is available at www.BioTechniques.com/article/114137.

Mitosis entails complex chromatin changes that have garnered increasing interest from biologists who study genome structure and regulation-fields that are being advanced by high-throughput sequencing (Seq) technologies. The application of these technologies to study the mitotic genome requires large numbers of highly pure mitotic cells, with minimal contamination from interphase cells, to ensure accurate measurement of phenomena specific to mitosis. Here, we optimized a fluorescence-activated cell sorting (FACS)based method for isolating formaldehyde-fixed mitotic cells-at virtually $100 \%$ mitotic purity and in quantities sufficient for high-throughput genomic studies. We compared several commercially available antibodies that react with mitosis-specific epitopes over a range of concentrations and cell numbers, finding antibody MPM2 to be the most robust and cost-effective.

Mitosis is characterized by drastic alterations to chromosome structure, global transcriptional silencing, and eviction of many transcriptional regulators from chromatin (1-3)(reviewed in Reference 4). Genome structure and regulation in the mitotic cell are emerging areas of research with important implications for understanding cellular memory of gene expression. What is the mechanism and function of the minority of transcriptional regulators that are retained at specific sites in the mitotic genome $(5,6,14,15)$ ? What are the dynamics of transcription factor occupancy, histone modifications, histone variants, and nucleosome positioning as cells traverse mitosis? How does mitotic chromosome condensation affect long-range chromosome interactions such as enhancer-promoter looping and large topological domains $(7,16)$ ? Researchers pursuing such questions increasingly rely on methods coupled to Seq technologies, such as chromatin immunoprecipitation (ChIP)-Seq, derivations of chromosome conformation capture (3C), and other epigenomic assays.

The application of such methods to study mitosis requires large numbers of pure mitotic cells to ensure sufficient signal-to-noise ratios and measurements that precisely reflect the state of mitotic cells, rather than contaminating interphase populations. ChIP studies comparing transcription factor occupancies in interphase and mitosis showed that, in some cases, mitotic occupancy occurred with reduced intensities and at only a fraction of interphase occupied sites $(5,6,14,15)$. In such situations, the purity of the mitotic population under study is critical for assessing whether the residual ChIP signals arise from true factor occupancy during mitosis, versus factor binding in a minority of contaminating interphase cells.

Meeting the stringent requirement for a high mitotic index can be challenging because only a small fraction $(<5 \%)$ of asynchronously growing cells are in mitosis. Cell cycle synchronization by pharmacologic treatments, such as nocodazole, can increase this percentage markedly, but in most cell types mitotic arrest is far from complete (Figure 1B)(5). In some adherent cell lines, enrichment of mitotic cells can be achieved by agitation of the tissue culture flask to detach loosely adhered mitotic cells (known as the mitotic shake-off method). However, this strategy fails in many adherent cell lines and primary cells, and is not applicable for suspension cells.

We and others previously developed a method to overcome these limitations by purifying mitotic cell populations using intracellular FACS. Cells were fixed as in ChIP experiments, permeabilized, and exposed to commercial antibodies against histone $\mathrm{H} 3$ phosphorylated at serine 10 (H3S10ph), a modification globally enriched during mitosis, to achieve $>98 \%$ pure mitotic cell populations $(5,8)$. Since developing this method, the original antibody used (clone MC463, 05-817; EMD Millipore, Billerica, MA) was discontinued. Subsequent

\section{Method summary:}

We surveyed and optimized reagents for FACS-assisted purification of mitotic cells. Of the antibodies surveyed, we found that MPM2 is $~ 100 \%$ specific, scalable, and comparatively cost-effective for purifying mitotic cells in bulk quantities suitable for high-throughput genomic studies. 
A Untreated
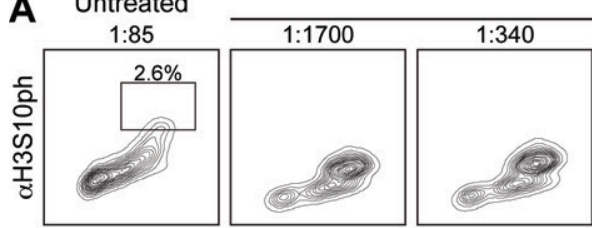

Nocodazole
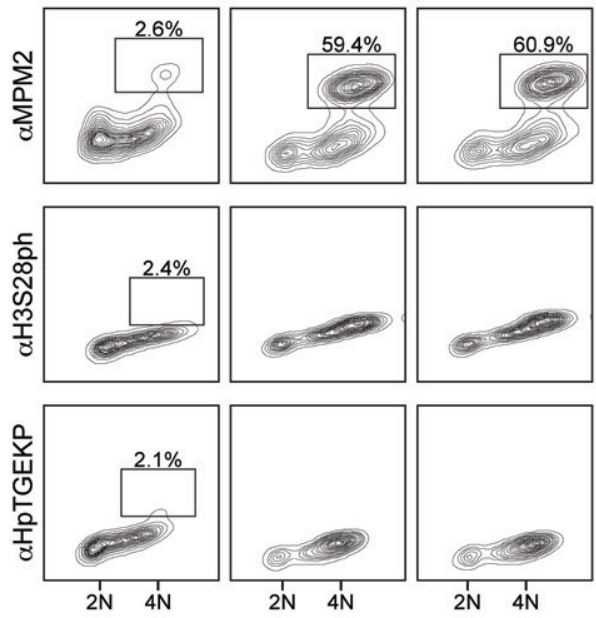

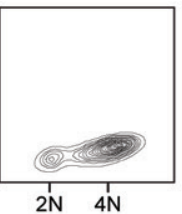

DNA content (PI)
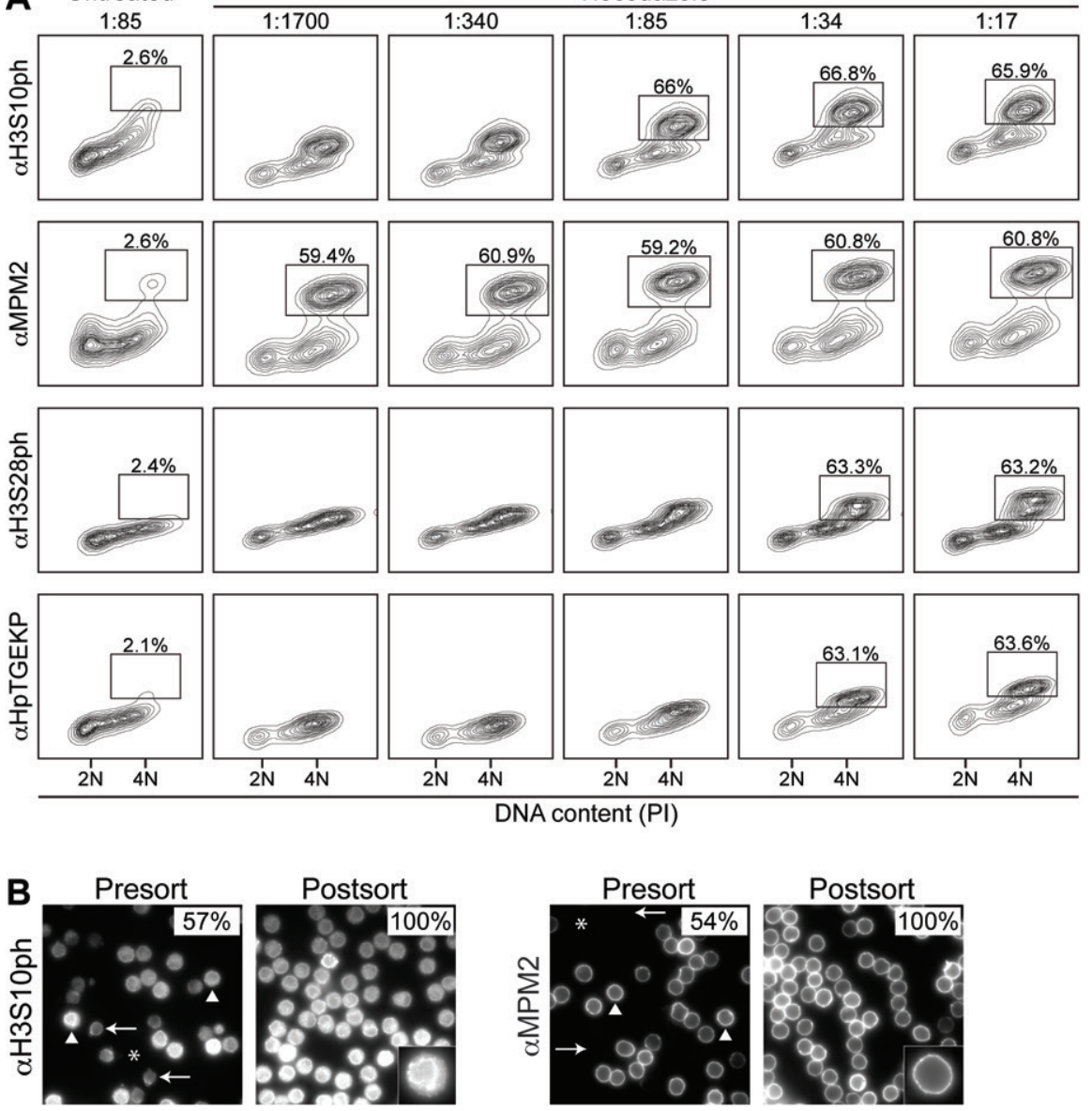

Postsort
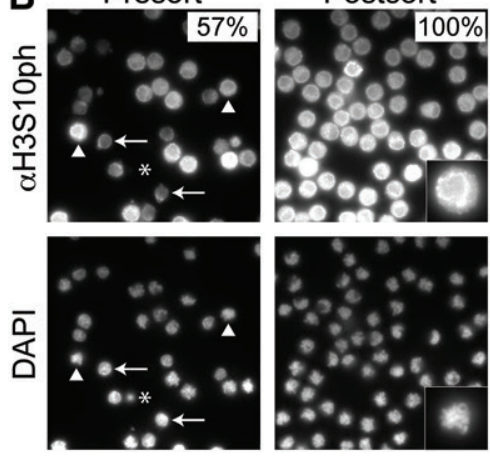
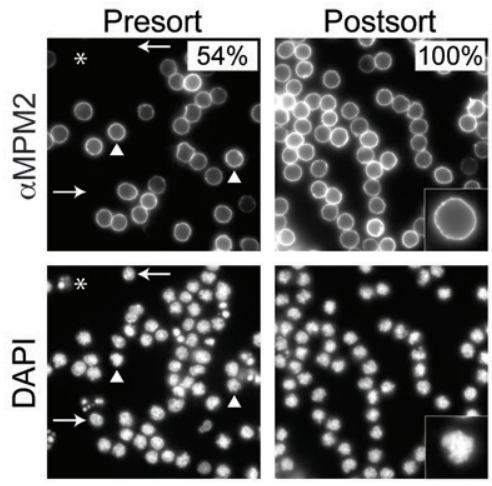

Figure 1. Titration of mitosis-specific antibodies for staining small numbers of murine cells.

(A) Ten million nocodazole (Sigma-Aldrich, St. Louis, MO)-treated (200 ng/mL for 8 h) or untreated asynchronous G1E cells were fixed in $1 \%$ formaldehyde (Thermo Fisher Scientific, Waltham, MA), permeabilized, resuspended at a density of $6 \times 10^{7}$ cells $/ \mathrm{mL}$, and stained with H3S10ph, MPM2, H3S28ph, or HpTGEKP antibodies at increasing doses (dilutions 1:1700, 1:340, 1:85, 1:34, 1:17) (see Supplementary Protocol for details). For nonfluorescent primary antibodies, Alexa Fluor 647-conjugated anti-rabbit IgG $\mathrm{F}\left(\mathrm{ab}^{\prime}\right)_{2}$ or allophycocyanin-conjugated anti-mouse IgG F(ab'), antibody fragments (Jackson ImmunoResearch, West Grove, PA) were used at a 1:85 dilution for detection. Propidium iodide (PI)(Sigma-Aldrich) was used to determine DNA content. For FACS plots where a separate mitotic population can be delineated by eye, a gate was drawn to indicate the percentage of positively staining cells. (B) Immunofluorescence microscopy of nocodazole-treated, crosslinked, and H3S10ph or MPM2 and DAPI stained G1E-ER cells before and after FACS confirms high purity of mitotic cells following cell sorting on a FACSAria II (Becton Dickinson, Franklin Lakes, NJ). Sorting by either H3S10ph ( $n=206$ cells) or MPM2 ( $n=161$ cells) staining yields populations of $100 \%$ mitotic cells as assayed by DAPI staining. Percentages indicate mitotic indices. Representative mitotic (arrowheads), interphase (arrows), and apoptotic (asterisks) cells are indicated. Insets: high magnification demonstrates unique H3S1Oph and MPM2 staining patterns. Imaging was carried out as follows: Samples were spun onto coverslips using a Shandon Cytospin Cytocentrifuge (Thermo Fisher Scientific). Single optical planes were taken on a Ti-E inverted fluorescence microscope (Nikon, Tokyo, Japan) using a 100x Plan-Apo objective (numerical aperture 1.42) and a cooled CCD camera (Pixis 1024B, Princeton Instruments, Trenton, NJ), using filter sets for DAPI (31000v2; Chroma, Bellows Falls, VT) and Alexa Fluor 647/APC (SP104v2; Chroma).

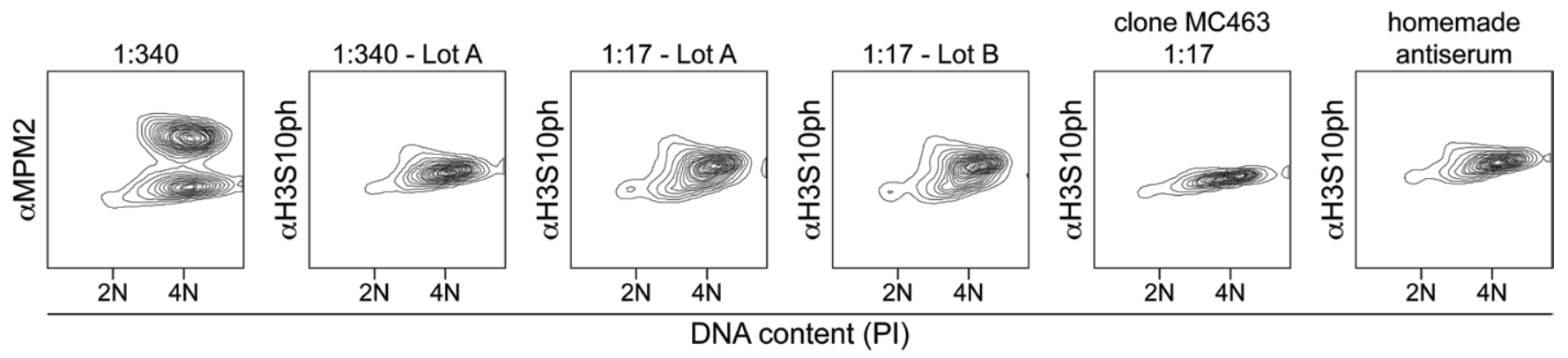

Figure 2. MPM2 outperforms H3S10ph when staining large numbers of murine cells. Two-hundred million G1E cells were treated with 200 $\mathrm{ng} / \mathrm{mL}$ nocodazole for $11 \mathrm{~h}$, formaldehyde-fixed, and stained with MPM2 or H3S10ph antibodies at 1:340 or 1:17 dilution at a constant cell density of $6 \times 10^{7}$ cells $/ \mathrm{mL}$ in the same fashion as in Figure 1. For the homemade H3S10ph antiserum, cells were completely bathed in $100 \%$ antiserum. Propidium iodide (PI) was used to determine DNA content. 
Table 1. Antibodies tested for ability to stain mitotic cells.

\begin{tabular}{|c|c|c|c|c|}
\hline Antibody & Abbreviation & Species, clonality & Source, catalog number & Epitope description \\
\hline $\begin{array}{l}\text { Anti-phospho Histone H3 } \\
\text { (Ser10), Mitosis Marker }\end{array}$ & H3S10ph & Rabbit, polyclonal & EMD Millipore, 06-570 and 04-817 & $\begin{array}{l}\text { Phosphorylated serine } 10 \text { on the N-terminal } \\
\text { tail of histone } \mathrm{H} 3 \text {; occurs during late } \mathrm{G} 2 \text { phase } \\
\text { to anaphase (12) }\end{array}$ \\
\hline $\begin{array}{l}\text { Anti-phospho-Ser/Thr-Pro, MPM-2 } \\
\text { (Mitotic protein monoclonal \#2) }\end{array}$ & MPM2 & Mouse, monoclonal & EMD Millipore, 05-368 & $\begin{array}{l}\text { Phosphorylated serines or threonines followed } \\
\text { by proline, specifically peptides LpTPLK or } \\
\text { FpTPLQ; occurs during mitosis }(9,13)\end{array}$ \\
\hline $\begin{array}{l}\text { Alexa Fluor } 647 \text { Rat anti-Histone } \\
\text { H3 (pS28) }\end{array}$ & H3S28ph & Rat, monoclonal & BD Biosciences, 558217 & $\begin{array}{l}\text { Phosphorylated serine } 28 \text { on the } \mathrm{N} \text {-terminal } \\
\text { tail of histone } \mathrm{H} 3 \text {; occurs during prophase to } \\
\text { anaphase (10) }\end{array}$ \\
\hline Anti-HpTGEKP motif & HpTGEKP & Rabbit, polyclonal & EMD Millipore, ABE319 & $\begin{array}{l}\text { Peptide HpTGEKP; based on the highly } \\
\text { conserved linker joining adjacent C2H2 zinc } \\
\text { fingers; occurs from prophase to anaphase (11) }\end{array}$ \\
\hline
\end{tabular}

batches scaled poorly in several attempts at purifying large quantities of mitotic cells (Supplementary Figure S1A). The reason for this lack of scalability remains unclear. Increasing the concentration of antibody from $1: 85$ to $1: 17$ when staining enough cells $\left(20 \times 10^{7}\right)$ for a single mitotic ChIP-Seq experiment failed to compensate for this impractically low staining efficiency of bulk quantities and raised the costs to $\geq \$ 760$ (at the current price of $\$ 380$ per vial). We raised our own antisera against the H3S10ph epitope, which proved similarly ineffective for staining bulk cell quantities (Supplementary Figure S1B). The lack of an effective scalable H3S10ph antibody is a major impediment to studying the mitotic epigenome.

We sought to improve the scalability and cost-effectiveness of FACS-based purification of formaldehyde-fixed mitotic cells by surveying three other commercially available antibodies against mitosis-specific epitopes: anti-phospho-Ser/Thr-Pro Mitotic protein monoclonal \#2 (MPM2)(9), anti-phospho Histone $\mathrm{H} 3$ (H3S28ph) (10), and anti-HpTGEKP motif (HpTGEKP)(11), and comparing them to the commercially available $\mathrm{H} 3 \mathrm{~S} 10 \mathrm{ph}$ antibody (12)(Table 1). First, we tested increasing concentrations of each for staining small numbers $\left(1 \times 10^{7}\right)$ of murine erythroid (G1E) suspension cells (Figure 1A) or human cervical cancer (HeLa) adherent cells (Supplementary Figure S2). For each antibody, a brightly staining population of mitotic cells with $4 \mathrm{~N}$ DNA content was detected. Separation between the dimly stained $4 \mathrm{~N}$ cells (S/G2 phase) and the brightly stained $4 \mathrm{~N}$ cells (mitosis) was achieved with $\geq 2 \mu \mathrm{L} / 1 \times 10^{7}$ cells of $\mathrm{H} 3510 \mathrm{ph}$ (1:85 dilution), $\geq 0.1 \mu \mathrm{L} / 1 \times 10^{7}$ cells of MPM2 (1:1700 dilution), and $\geq 5 \mu \mathrm{L} / 1 \times$ $10^{7}$ cells of H3S28ph or HpTGEKP (1:34 dilution). At all tested concentrations, MPM2 achieved the clearest separation between dim and bright $4 \mathrm{~N}$ cells. Notably, cells stained with the lowest concentration of MPM2 had better separation than any other antibody used at the highest concentration. Therefore, MPM2 is the most effective antibody for staining mitotic cells in relatively small numbers, followed by H3S10ph. In these small scale experiments, both MPM2- and H3S10ph-based FACS yielded $\sim 100 \%$ mitotic cells as determined by post-sort immunofluorescence and DAPI (Molecular Probes, Eugene, OR) counterstaining (Figure 1B). We note that while $\mathrm{H} 3 \mathrm{~S} 10 \mathrm{ph}$ coats mitotic chromatin, MPM2 staining is en riched at the plasma membrane.

We next compared the performance of $\mathrm{H} 3 \mathrm{~S} 10 \mathrm{ph}$ and MPM2 in staining large numbers $\left(20 \times 10^{7}\right)$ of G1E cells, a quantity commonly required for ChIP-Seq and 3C-based assays. Such high starting cell numbers seem excessive but are necessary because a fraction of formaldehyde-fixed cells are lost from sticking to various surfaces during sample processing. When used at $0.5 \mu \mathrm{L} / 1 \times 10^{7}$ cells (1:340 dilution), MPM2 resolves a clear mitotic population (Figure 2), maintaining a similar staining pattern to that seen with small cell numbers (Figure 1A). In contrast, when used at concentrations from $0.5 \mu \mathrm{L} / 1 \times 10^{7}$ cells to 10 $\mu \mathrm{L} / 1 \times 10^{7}$ cells ( $1: 340$ to $1: 17$ dilution), H3S10ph fails to clearly identify a mitotic sub-population. Similar results were obtained with an independent lot of $\mathrm{H} 3 \mathrm{~S} 10 \mathrm{ph}$ antibody, the MC463 clone (04-817, EMD Millipore), and our homemade $\mathrm{H} 3 \mathrm{~S} 10 \mathrm{ph}$ antiserum (Figure 2). Prolonged incubation at $4^{\circ} \mathrm{C}$ did not improve $\mathrm{H} 3 \mathrm{~S} 10 \mathrm{ph}$ staining (data not shown).

In summary, MPM2 outperforms other tested mitosis-specific antibodies in large-scale purifications of mitotic cells.Our data show that, of the antibodies surveyed, MPM2 is highly specific for mitotic cells, and is currently the only scalable and cost-effective antibody for isolating bulk preparations of pure mitotic cells by FACS. At current antibody prices, a typical experiment to stain $20 \times 10^{7}$ cells would cost $\$ 20$ in MPM2 antibody (using a 1:340 dilution), compared with $\geq \$ 760$ in H3S10ph antibody (using a 1:17 dilution that still fails to robustly identify mitotic cells). The MPM2 antibody-based method is a substantial improvement in scalability and cost for FACS-purifying cells for use in ChIP-Seq, 3C-based, and other assays requiring large quantities of starting material. Our protocol should be generally applicable to any cell line, primary cells, or tissue samples amenable to FACS. Commercial antibodies may vary between lots as we observed for H3S10ph antibodies. Therefore, it might be necessary to recalibrate the staining protocol, for example by varying parameters such as detergent concentrations, incubation times, and antibody concentrations. In the future, adaptation of this protocol may enable the analysis of RNA or protein species in pure populations of mitotic cells.

\section{Author Contributions}

A.E.C and C. C.-S. H. conceived the study, designed and performed experiments, and analyzed data. A.E.C, C. C.-S. H., and G.A.B. wrote the manuscript.

\section{Acknowledgments}

The authors thank Suzanne N. Shapiro for technical support and Arjun Raj for use of his microscope. This study was supported by grants from the National Institutes of Health [5R01DK054937 
(G.A.B.), T32DK07780 (A.E.C.) and T32GM008216 (C. C.-S. H.)]. This paper is subject to the NIH Public Access Policy.

\section{Competing Interests}

The authors declare no competing interests.

\section{References}

1. Taylor, J.H. 1960. Nucleic acid synthesis in relation to the cell division cycle. Ann. N. Y. Acad. Sci. 90:409-421.

2. Prescott, D.M. and M.A. Bender. 1962. Synthesis of RNA and protein during mitosis in mammalian tissue culture cells. Exp. Cell Res. 26:260-268.

3. Martínez-Balbás, M.A., A. Dey, S.K. Rabindran, K. Ozato, and C. Wu. 1995. Displacement of sequence-specific transcription factors from mitotic chromatin. Cell 83:29-38.

4. Kadauke, S. and G.A. Blobel. 2013. Mitotic bookmarking by transcription factors. Epigenetics Chromatin 6:6.

5. Kadauke, S., M.I. Udugama, J.M. Pawlicki, J.C. Achtman, D.P. Jain, Y. Cheng, R.C. Hardison, and G.A. Blobel. 2012. Tissuespecific mitotic bookmarking by hematopoietic transcription factor GATA1. Cell 150:725-737.

6. Caravaca, J.M., G. Donahue, J.S. Becker, X. He, C. Vinson, and K.S. Zaret. 2013. Bookmarking by specific and nonspecific binding of FoxA 1 pioneer factor to mitotic chromosomes. Genes Dev. 27:251-260.

7. Deng, W. and G.A. Blobel. 2010. Do chromatin loops provide epigenetic gene expression states? Curr. Opin. Genet. Dev. 20:548-554.

8. Follmer, N.E. and N.J. Francis. 2012. Preparation of Drosophila tissue culture cells from different stages of the cell cycle for chromatin immunoprecipitation using centrifugal counterflow elutriation and fluorescence-activated cell sorting. Methods Enzymol. 513:251-269.

9. Davis, F.M., T.Y. Tsao, S.K. Fowler, and P.N. Rao. 1983. Monoclonal antibodies to mitotic cells. Proc. Natl. Acad. Sci. USA 80:2926-2930.

10. Goto, H., Y. Tomono, K. Ajiro, H. Kosako, M. Fujita, M. Sakurai, K. Okawa, A. Iwamatsu, et al. 1999. Identification of a novel phosphorylation site on histone $\mathrm{H} 3$ coupled with mitotic chromosome condensation. J. Biol. Chem. 274:25543-25549.

11. Rizkallah, R., K.E. Alexander, and M.M. Hurt. 2011. Global mitotic phosphorylation of $\mathrm{C} 2 \mathrm{H} 2$ zinc finger protein linker peptides. Cell Cycle 10:3327-3336.

12. Hendzel, M.J., Y. Wei, M.A. Mancini, A. Van Hooser, T. Ranalli, B.R. Brinkley, D.P. Bazett-Jones, and C.D. Allis. 1997. Mitosis-specific phosphorylation of histone H3 initiates primarily within pericentromeric heterochromatin during G2 and spreads in an ordered fashion coincident with mitotic chromosome condensation. Chromosoma 106:348-360.
13. Westendorf, J.M., P.N. Rao, and L. Gerace. 1994. Cloning of cDNAs for M-phase phosphoproteins recognized by the MPM2 monoclonal antibody and determination of the phosphorylated epitope. Proc. Natl. Acad. Sci. USA 91:714-718.

14. Follmer, N.E., A.H. Wani, and N.J. Francis. 2012. A polycomb group protein is retained at specific sites on chromatin in mitosis. PLoS Genet. 8:e1003135.

15. Yang, J., E. Sung, P.G. Donlin-Asp, and V.G. Corces. 2013. A subset of Drosophila Myc sites remain associated with mitotic chromosomes colocalized with insulator proteins. Nat. Commun. 4:1464.

16. Naumova, N., M. Imakaev, G. Fudenberg, Y. Zhan, B.R. Lajoie, L.A. Mirny, and J. Dekker. 2013. Organization of the mitotic chromosome. Science 342:948-953.

Received 01 December 2013; accepted 06 January 2014.

Address correspondence to Gerd A. Blobel, The Children's Hospital of Philadelphia, Philadelphia, PA. E-mail: blobel@email. chop.edu

To purchase reprints of this article, contact: biotechniques@fosterprinting.com 